\title{
Surface-plasmon-enhanced light transmission through planar metallic films
}

\author{
L. Lin ${ }^{1}$, R. J. Reeves ${ }^{2}$ and R. J. Blaikie ${ }^{1, *}$ \\ ${ }^{1}$ MacDiarmid Institute for Advanced Materials and Nanotechnology, Department of \\ Electrical and Computer Engineering, University of Canterbury, Christchurch 8020, New \\ Zealand \\ ${ }^{2}$ MacDiarmid Institute for Advanced Materials and Nanotechnology, Department of \\ Physics and Astronomy, University of Canterbury, Christchurch 8020, New Zealand \\ *Corresponding author, e-mail: r.blaikie@elec.canterbury.ac.nz
}

\begin{abstract}
We show experimental results for the optical transmission of planar metallic films closely coupled to periodic sub-wavelength structures; exceptionally-high transmission levels (up to $39 \%$ ) are measured for these otherwise-opaque films. The measured dispersion of the transmission maxima agrees well with the theoretical dispersion of surface plasmon polariton (SPP) modes on a planar metal film, which strongly suggests that the excitation of these SPP modes is responsible for the exceptional transmission features. This is further supported by rigorous coupled wave analysis (RCWA) simulations. The overall transmission is a result of the coupling between the incident light and the SPP modes, as well as the localized surface plasmon (LSP) modes within the structure.
\end{abstract}

PACS numbers: $\{71.36 .+\mathrm{c} ;$ 73.20.Mf; 78.66.-w; 42.79.Dj\} Accepted for publication in Phys Rev B (Received 28 May 2006, Accepted 11 August 2006) 
Enhanced optical transmission through thin metallic films with periodic subwavelength structures has attracted a great deal of interest in recent years. The phenomenon has been observed on various structures $[1,2,3]$ and ,in addition multilayer sub-wavelength metallic structures have also been measured to have bands where negative-refractive-index behaviour is observed [4,5], adding to their current interest. It is now well accepted that the excitation of surface plasmons (SPs) plays an important role in the extraordinary transmission through sub-wavelength hole arrays, however the fact that the SPs and the sub-wavelength features that generate them co-exist on the same film (or films) tends to confound the analysis somewhat. As a result, many analytical models $[6,7,8,9,10,11,12]$ have been proposed to understand their physical origins.

While most works in this area have been concentrated on perforated metallic films, the concept of SP-assisted light tunneling has been presented based on the studies of the corrugated [3] and smooth metal films [13]. Subsequently, some authors have suggested that continuous metallic films can also exhibit enhanced transmission provided they are periodically modulated $[10,11,12,14]$. Extending this further, it is worthwhile to also consider the transmission characteristics of completely planar metal films (no modulation whatsoever) with some independent means for SP excitation. In this letter, we present an experimental study of the optical properties of such unpatterned planar metallic films closely coupled to periodic structures for SP excitation, showing that it is not necessary to have surface modulation on or through the metal film to achieve the enhanced transmission; the planar films can also give rise to the extraordinary transmission so long as there is some means of generating the necessary SP modes. The mechanisms involved here are somewhat different to an earlier report on obtaining high transparency of metal films via symmetrical arrangement of prism couplers on opposite of metal surfaces [15]; the 
structure employed in this study enables the decoupling of the SP generation from the metal film itself, and thus provides important insights into the nature of the enhanced transmission effects.

A schematic of the structure we have studied experimentally is shown in Fig. 1. It consists of a glass substrate with a thin planar silver (Ag) film, a dielectric spacer and another thin Ag film perforated with subwavelength hole arrays on top. The thickness of this hole-array layer is much less $(35 \mathrm{~nm})$ than that in previous reported studies $[1,8]$. Its primary roles are to match the momentum of the incident light and SPPs and to induce the required surface charge distribution on the planar Ag film underneath through near-field coupling from the evanescent orders of the light diffracted by the subwavelength hole arrays [16]. Using this configuration allows us to easily identify the origins of different modes by analyzing the spectral response of the structure to the variations of its geometric arrangement and the excitation condition.

The sample fabrication process involves vacuum deposition of thin films $\left(\mathrm{Ag}\right.$ and $\left.\mathrm{SiO}_{2}\right)$, double-exposure interference lithography for pattern definition, reactive ion etching and lift-off for pattern transfer. The details of the procedure can be found elsewhere [17]. All the fabricated samples have a $33 \mathrm{~nm}$-thick planar $\mathrm{Ag}$ film, a $195 \mathrm{~nm}$-thick $\mathrm{SiO}_{2}$ layer and a $35 \mathrm{~nm}$-thick hole-array layer. The lattice constants of the hole arrays in two orthogonal directions $\left(P_{x}\right.$ and $\left.P_{y}\right)$ are slightly $(\sim 10 \mathrm{~nm})$ mismatched by design on the sample. The range of the lattice constants on the samples varies from $460 \mathrm{~nm}$ to $560 \mathrm{~nm}$. A scanning electron microscope (SEM) image of the hole-array layer on one of the fabricated samples is shown in Fig. 1(b). 
We analyzed the zeroth order transmission and reflection spectra of the structure under linearly polarized light. The measurements were made over the wavelength range $550 \mathrm{~nm}$ to $1000 \mathrm{~nm}$ at $1 \mathrm{~nm}$ intervals with a bandwidth of $0.5 \mathrm{~nm}$. Figure 2(a) shows the measured transmission spectra of a typical sample at normal incidence. The sample is fabricated on BK7 glass substrate with $P_{x}=460 \mathrm{~nm}$ and $P_{y}=470 \mathrm{~nm}$, on which the holes have an elliptic shape with axis lengths $D_{x}=238 \mathrm{~nm}$ and $D_{y}=216 \mathrm{~nm}$ in the $x$ - and $y$-directions, respectively. The dashed and solid lines represent the transmissions under which the electric field of the incident light oscillates parallel with $P_{x}$ and $P_{y}$ respectively. The corresponding simulation results obtained from a rigorous coupled-wave analysis (RCWA) [18] based commercial grating analysis software, G-Solver [19], are shown in Fig 2(b). In the simulation, the elliptic holes are replaced with rectangular holes of the same surface area to reduce computation time. The complex refractive indices of Ag are attained from linear interpolation of the tabulated data in Ref [20]; the refractive indices of the $\mathrm{SiO}_{2}$ and BK7 glass are set to 1.454 and 1.512 , respectively.

In Fig. 2, we observe a very sharp, period-dependent transmission peak (peak 2) in the transmission spectrum, followed by a smaller peak (peak 3). The measured transmission maximum reaches $39 \%$ in Fig. 2(b). To investigate the relationship between the spectral positions of these peaks and the lattice constant of the hole arrays, we fabricated a set of samples with different lattice constants on fused silica substrates. The measured spectral positions of the second and the third peaks at normal incidence versus the lattice constants of the hole arrays are displayed in Fig. 3. Using the fused silica minimizes the unwanted effects caused by the asymmetric arrangement of materials surrounding the Ag film, even though both experiments and simulations show that a slight mismatch between the dielectric constants of substrate and spacer (e.g., BK7 glass and fused silica) produces 
higher transmission than the symmetric arrangement. For a thin metal film $(<100 \mathrm{~nm})$ bounded by same dielectric medium on two surfaces, SPP waves of same frequencies on two interfaces of the metal film will interfere, resulting in a splitting of SPPs into a high frequency $\omega^{+}$mode and a low frequency $\omega^{-}$mode. The implicit dispersion relation for these two modes can be expressed as [21]

$$
\begin{aligned}
& \omega^{+}: \varepsilon_{m} k_{z d}+\varepsilon_{d} k_{z m} \tanh \frac{k_{z m} l}{2 i}=0, \\
& \omega^{-}: \varepsilon_{m} k_{z d}+\varepsilon_{d} k_{z m} \operatorname{coth} \frac{k_{z m} l}{2 i}=0,
\end{aligned}
$$

where: $\varepsilon_{m}$ and $\varepsilon_{d}$ are the dielectric constants of the metal and the surrounding dielectrics, respectively; $l$ is the thickness of the metal film; and $k_{z m}$ and $k_{z d}$ are wavevectors in the metal and the dielectric with direction normal to the interface, respectively. The spectral positions of these two modes excited by a periodic structure with lattice constants $p$ at normal incidence can be calculated by using the following relationships:

$$
\begin{aligned}
& \left(a k_{s p}\right)^{2}+k_{z i}^{2}=\varepsilon_{i}\left(\frac{\omega}{c}\right)^{2}, i=m, d \\
& a k_{s p}=m \frac{2 \pi}{p}
\end{aligned}
$$

where $a$ is a frequency-dependent constant that is chosen to solve the equation (1) or (2) numerically, $k_{s p}$ is the wavevector of SPPs along a semi-infinite metal surface $\left(k_{s p}=\frac{\omega}{c} \sqrt{\frac{\varepsilon_{d} \varepsilon_{m}}{\varepsilon_{m}+\varepsilon_{d}}}\right.$ ) and $m$ is an integer for the mode index. The calculated dispersion relations of $\omega^{ \pm}$modes on the interfaces of a $33 \mathrm{~nm}$-thick Ag film surrounded by semiinfinite $\mathrm{SiO}_{2}$ layers are also plotted in Fig. 3: the dashed line and the solid line represent the calculated $\omega^{+}$and the $\omega^{-}$mode, respectively. In addition, the SPP mode on the interface of a semi-infinite Ag layer is also displayed (dotted line) in Fig. 3. We observe that for samples with higher transmissions (lattice constants $500 \mathrm{~nm}-530 \mathrm{~nm}$, where transmission 
of the second peak is $>20 \%$ ), the measured spectral positions agree well with the theoretical calculations. This strongly suggests that the second and the third peak in the transmission spectra are attributable to the excitation of $\omega^{+}$and $\omega^{-}$mode on the planar Ag film.

For those samples with low SPP-enhanced transmission (lattice constants less than $500 \mathrm{~nm}$ and peak transmission $<20 \%$ ), the SPP related modes show red shifts in the measured frequencies and they exhibit an opposite trend while the lattice constants become larger than the optimal values. This is due to the fact that the calculation above includes only interaction between two SPP modes on the surfaces of the planar Ag films; it does not consider any other coupling effects that might exist within the structure. In fact, the overall transmission of the structure involves coupling between the incident light, SPP modes, LSP modes and the cavity mode; we will discuss this in detail after verifying the origin of each peak appearing in the transmission spectra.

We analyzed the spectral response of the sample under different excitation conditions. Fig. 4 shows the zeroth order transmission spectra of a sample $\left(P_{x}=501 \mathrm{~nm}, P_{y}=513 \mathrm{~nm}\right.$, $D_{x}=240 \mathrm{~nm}$ and $D_{y}=238 \mathrm{~nm}$, substrate material: fused silica) under TE- (solid line) and TM-polarized (dashed line) light at 5 degrees of incidence with the plane of incidence along the $x$-direction of Fig. 1. Unsurprisingly the shape of the transmission spectrum changes noticeable under TM-polarized light due to the extra momentum the SPPs gain from the incident light at oblique incidence. Conversely, under TE-polarized light the SPP related transmission peaks remain in the spectrum while varying the angle of incidence. While this seems to contradict the nature of SPPs at the first sight, it can be explained by considering the excitation of the localized charge oscillations (and subsequently the near- 
field enhancement) by the incident light at the edges of the holes [16] - the enhanced electric field extends to the planar Ag film underneath and induces the required surface charges to launch SPP waves on the planar film. Since the wavevector of the incident light and the oscillations of the E-field are out of phase for TE-polarized light, the wavelength of the SPP modes are unaffected by the incident light and solely depends on the lattice constant $P_{y}$. This unique feature provides us a useful tool to analyze the reflection and absorption response of the sample since in practise it is difficult to measure the reflection spectrum at normal incidence. Figure 5 shows the zeroth order transmission, reflection and absorption spectra while illuminating the sample from the hole-array side (dashed lines) and the substrate side (solid lines) under TE-polarized light at 5 degrees angle of incidence. The absorption spectra were obtained via $A=1-T-R$, where $A, T$ and $R$ are the absorbance, transmittance and reflectance respectively. Clearly the transmission of the structure is independent of which side the sample is illuminated; in contrast, the reflection and the absorption spectra are rather different for light incident from the hole-array side or from the substrate side. This observation agrees with the recent reports $[12,22]$ : owing to internal damping of the SPs inside the metal in the process of SP-enhanced transmission, enhanced absorption also exists; the absorption is related to the SPP excitation efficiency hence is sensitive to the illumination condition. While illuminating from the hole-array side, the reflectance of both $\omega^{+}$and $\omega^{-}$modes are very low, which indicates excellent excitation efficiency of the SPPs on the planar Ag film under this arrangement. The divergence in the transmittance of $\omega^{+}$and $\omega^{-}$modes is due to the fact that the electric fields associated with these two modes inside the metal film are different - the electric field related to the $\omega^{+}\left(\omega^{-}\right)$mode inside the film decreases (increases) as the film thickness reduces [21]. For the thickness of the planar film studied here, the rate of the internal damping of the $\omega^{+}$mode is smaller than that of the $\omega^{-}$mode, resulting in the lower 
absorption, higher transmission efficiency and narrower linewidth of this mode. While illuminating from the substrate side, the reflectance of both $\omega^{+}$and $\omega^{-}$modes increase, particularly the $\omega^{-}$mode. This suggests that the excitation of SPPs on the interfaces of the planar film are weaker under this arrangement, subsequently the interaction of two SPPs existing on the two interfaces declines, resulting in imbalanced coupling efficiency of $\omega^{+}$ and $\omega^{-}$modes.

There are two relatively broad transmission peaks that appear in the transmission spectra of the sample, one situated below of the $\omega^{+}$mode (peak 1 in Fig. 2) and the other one located beyond the $\omega^{-}$mode (peak 4 in Fig. 2). From Figs. 4 and 5, one notices that varying the polarization state of the incident light also alters the shape of peak 1 and changing the illumination condition has little affect on its feature in reflection and absorption spectra. The results from measurements and simulations (not shown here) reveal that the spectral position and amplitude of this peak are highly sensitive to the dimensions of the holes and the thickness and optical constants of the dielectric spacer, which are the characteristics of waveguide modes. The change in the position of the peak 1 in Fig. 2 while varying the orientation of the electric field of incident light is mainly due to the asymmetric shape of the holes on the sample. Simulations confirm that the orientation of the electric field of the incident light only affects the features of this peak if the holes are rectangular in shape; it has no effect on square holes. We hence conclude that peak 1 arises from the excitation of a waveguide mode within the structure. On the other hand, peak 4 exhibits both SPP and LSP nature. It is to be noticed in Fig. 2 that the position of peak 4 also shows a hole-arrayperiod dependency. Its reflectance and the absorbance strongly depend on the illumination side of the sample in Fig. 5. However, compared with peak 2 and peak 3 it is much less sensitive to the polarization state of incident light under oblique incidence. From 
simulations, the characteristics of this peak are closely related to the thickness of the dielectric spacer, as well as the dimensions of the holes (more precisely, the separation between them). Therefore we consider this peak to have a "mixed mode" characteristic.

The effects of the hole size on the transmittance and the reflectance of the structures have also been studied via simulation, as show in Fig. 6: in Fig. 6(a), $P_{x}=P_{y}=490 \mathrm{~nm}, D_{x}=$ $0.35 P_{x}$ and $D_{y}=0.35 P_{y}$; in Fig. 6(b), $P_{x}=P_{y}=490 \mathrm{~nm}, D_{x}=0.5 P_{x}$ and $D_{y}=0.5 P_{y}$; in Fig. 6(c), $P_{x}=400 \mathrm{~nm}, P_{y}=490 \mathrm{~nm}, D_{x}=0.5 P_{x}$ and $D_{y}=0.5 P_{y}$. The substrate is $\mathrm{SiO}_{2}$ and the Efield of the normally incident light is along $y$-direction. Clearly reducing the surface area of the hole boosts the strength of SPP related transmission peaks (peaks 2 and 3), however this also broadens their linewidth and introduces blue shifts in their spectral positions. We regard this as a consequence of coupling between the LSP mode and the SPP modes within the structure. In periodic subwavelength structures, the observed LSP excitation is indeed a result of the interaction between LSPs on the individual elements of the structure [23]. Therefore, varying the geometry of holes modifies both the frequencies and the strength of the LSP mode. A recent work showed that in the region where the frequencies of the LSP mode and the SPP mode are close together, coupling between the two modes can bring about extraordinary optical transmission for metallic films perforated with periodic subwavelength structures [24]. A similar effect is observed here for our planar metal film structures.

To further investigate the mechanism behind the exceptional transmission observed in the structures studied here, we systematically analyzed the expected transmission and the absorption of the structures with lattice constants of the hole array range from $300 \mathrm{~nm}$ to $900 \mathrm{~nm}$ with a step size of $10 \mathrm{~nm}$. Fig. 7 shows one set of simulation results. Here $P_{y}$ 
ranges from $300 \mathrm{~nm}$ to $900 \mathrm{~nm}$ and $D_{y}=0.5 P_{y}\left(\mathbf{E} / / P_{y}\right) ; P_{x}$ and $D_{x}$ are fixed to $400 \mathrm{~nm}$ and $200 \mathrm{~nm}$, respectively. As the critical value for excitation of waveguide mode through the holes is $D>\lambda / 2 n^{2}$ ( $n=1$ for air here) in the direction perpendicular to the E-field, this configuration suppresses the waveguide mode while maintaining the excitation efficiency of SPP modes for the incident light with wavelengths larger than $400 \mathrm{~nm}$. The calculated dispersion relation the of $\omega^{+}$and $\omega^{-}$modes on the thin Ag films via Eq. (1) and Eq.(2) are also shown as the dashed and the solid lines respectively. We notice that the spectral positions of two transmission (and absorption) peaks in each individual simulation closely follow these two lines, which further confirms the observed peaks derived from SPPs.

In Fig. 7, there are two hole-arrays-lattice-constant independent modes at $\omega_{1} \approx$ $4.10 \times 10^{15} \mathrm{rad} / \mathrm{s}\left(\lambda_{1}=460 \mathrm{~nm}\right)$ and $\omega_{2} \approx 2.75 \times 10^{15} \mathrm{rad} / \mathrm{s}\left(\lambda_{2}=685 \mathrm{~nm}\right)$. The high frequency one shows very low transmission and very high absorption. In contrast, both the transmission and the absorption of the low frequency mode are relatively low compared with its neighbourhood. The phase shifts of these two modes after they each travel a round trip within the dielectric spacer can be calculated via $\theta=4 \pi n_{d} d / \lambda$, where $n_{d}$ and $d$ are the refractive index and the thickness of the dielectric spacer respectively. This yields $\theta_{1} \approx 2 \frac{1}{2} \pi$ and $\theta_{2} \approx 1 \frac{1}{2} \pi$. Considering there is a phase difference of $1 / 2 \pi$ between the propagating waves and the evanescent waves, it is clear that $\omega_{1}$ mode arises from the constructive interference between the incident light and the evanescent modes existing in the dielectric spacer, which increases the absorbance of the structure. The $\omega_{2}$ mode results from the destructive interference between the incident light and the evanescent modes. This destructive cavity mode imposes a band gap across the $\omega^{+}$and $\omega^{-}$modes and causes the bending of the SPP modes at the edge of the gap, as highlighted by " $\mathrm{B}$ " and " $\mathrm{C}$ " in Fig. 7(b). This explains the discrepancy between the measured and calculated spectral 
positions of $\omega^{+}$and $\omega^{-}$in Fig. 3 for samples with smaller lattice constants $(P: 460 \mathrm{~nm} \sim$ $\left.480 \mathrm{~nm} ; k_{s p}: \sim 1.3 \times 10^{-7} \mathrm{~m}^{-1}\right)$. The LSP mode existing in the hole-array layers is labelled "A" in Fig. 7(b). This mode also appears on the dispersion map of the structures that consist of a hole-array-only layer and its spectral response shows high $A$, low $R$ and moderate $T$. The shape of this mode in the dispersion diagram is governed by the dimensions of the holes for both hole-array-only and multi-layer structures. This mode interacts with the destructive cavity mode, with its tail situated just above $\omega_{2}$. Nevertheless, its spectral response primarily shows the characteristics of the guided mode (peak 1 in Fig. 2). The mode with label " $\mathrm{D}$ " in Fig. 7 is inconspicuous on the hole-array-only structure; this mode arises from the interaction of the waveguide mode and LSP modes (peak 4 in Fig. 2). However, the strength of the LSP mode in this region is relatively weak compared to that in region "A". Decreasing the surface area of the holes will strengthen LSPs in this region (Fig. 6), but in general their effects on the enhanced transmission are insubstantial.

We observed that high transmission efficiency of the structure only occurs in the vicinity where the LSP mode intersects the SPP modes. This agrees with the suggestion of enhanced transmission being the result of resonant SP-assisted light tunneling through metal films $[10,11]$. The resultant spectra are subject to the strength of each mode. If SPP modes dominate the interaction we have very sharp, lattice-constant-dependent transmission peaks with their spectral positions well matching the dispersion relation of SPPs on the planar metal films. Intensifying the effects of the LSP mode will broaden the linewidth of the SPP peaks, reducing their amplitude and moving them to the high frequency side of the spectrum. This gives the explanation for the blue shifts observed in the measured transmission spectra for samples with larger lattice constants $(P>530 \mathrm{~nm})$. 
The transmittance of the structure fades away in the region where the LSP and the SPP modes become isolated. The crucial difference between SPP enhanced transmission observed in the structure studied in this paper and that in metal films perforated with subwavelength hole arrays lays in the characteristics of the SPP peaks. For the structure studied here, the linewidth of the transmission peak is much narrower and its centre wavelength can be precisely controlled by adjusting the geometric arrangement of the structure, which offers great potential in developing surface-plasmon-based optical devices.

In conclusion, we have demonstrated that the enhanced optical transmission can be achieved through the planar metallic films by closely coupled the films to subwavelength hole arrays. The results from the experiments and the theoretical calculations confirm that the exceptional transmission is due to excitation of surface plasmon polariton modes on two interfaces of the planar metal films. The overall transmission property of the structure is a result of the couplings between the incident light, SPP modes and LSP modes within the structure. The interaction between SPPs and LSP gives rise to the exceptional transmission through planar metal films and the transmission efficiency fades away in the regions where either of these modes is absent.

The authors wish to thank Helen Devereux and Gary Turner for their technical support, Dr. Damian Carder for assistance with UV laser and John Hamlin for assistance with optical measurements. 


\section{References}

1 T. W. Ebbesen, H. J. Lezec, H. F. Ghaemi, T. Thio, and P. A. Wolff, Nature 391, 667 (1998).

2 H. J. Lezec, A. Degiron, E. Devaux, R. A. Linke, L. Martin-Moreno, F. J. GarciaVidal, and T. W. Ebbesen, Science 297, 820 (2002).

3 I. Avrutsky, Y. Zhao, and V. Kochergin, Optics Letters 25, 595 (2000).

4 V. M. Shalaev, W. S. Cai, U. K. Chettiar, H. K. Yuan, A. K. Sarychev, V. P. Drachev, and A. V. Kildishev, Optics Letters 30, 3356 (2005).

5 S. Zhang, W. J. Fan, N. C. Panoiu, K. J. Malloy, R. M. Osgood, and S. R. J. Brueck, Physical Review Letters 95, 137404 (2005).

6 J. A. Porto, F. J. Garcia-Vidal, and J. B. Pendry, Physical Review Letters 83, 2845 (1999).

7 E. Popov, M. Neviere, S. Enoch, and R. Reinisch, Physical Review B 62, 16100 (2000).

8 L. Martin-Moreno, F. J. Garcia-Vidal, H. J. Lezec, K. M. Pellerin, T. Thio, J. B. Pendry, and T. W. Ebbesen, Physical Review Letters 86, 1114 (2001).

$9 \quad$ M. M. J. Treacy, Physical Review B 66, 195105(2002).

10 D. Gerard, L. Salomon, F. de Fornel, A. V. Zayats, Optics Express, 12, 3652 (2004).

11 S. A. Darmanyan, M. Nevière, and A. V. Zayats, Physical Review B 70, 075103, (2004).

12 D. Gerard, L. Salomon, F. de Fornel, and A. V. Zayats, Physical Review B 69, 113405 (2004). 

(2003). (2003). (1985). B 68, 205103 (2003).

17 L. Lin, R. J. Blaikie, and R. J. Reeves, Journal of Electromagnetic Waves and Applications 19, 1721 (2005). Optical Society of America a-Optics Image Science and Vision 12, 1068 (1995).

19 http://www.gsolver.com. P. B. Johnson and R. W. Christy, Physical Review B 6, 4370 (1972).

H. Raether, Surface plasmons on smooth and rough surfaces and on gratings (Berlin ; New York : Springer-Verlag, 1988). Review Letters 92, 107401 (2004). (2005).

A. Degiron and T. W. Ebbesen, Journal of Optics A, 7, 90 (2005). 


\section{Figure Captions}

Figure 1. (a) A schematic diagram of the structure studied. (b) A SEM image of a fabricated hole-array layer.

Figure 2. (Color on line) Transmission spectra of a fabricated sample at normal incidence with the E-field parallel to $P_{x}(----)$ and $P_{y}(-)$ : (a) measurements; and (b) the corresponding simulation results.

Figure 3. (Color on line) Dispersion relations of the $2^{\text {nd }}$ and the $3^{\text {rd }}$ peaks for samples with hole-array periods varying from 450 to $560 \mathrm{~nm}$. Also shown are the theoretical single-interface SPP line and the $\omega^{+}$and $\omega^{-}$lines.

Figure 4. (Color on line) Transmission spectra of a sample with $P_{x}=501 \mathrm{~nm}$ and $P_{y}=513$ nm under TE- ( - ) and TM-polarized (----) light at 5 degrees angle of incidence.

Figure 5. (Color on line) (a) Transmission, (b) reflection and (c) absorption spectra of a sample with $P_{x}=501 \mathrm{~nm}$ and $P_{y}=513 \mathrm{~nm}$ while illuminating from the hole-array (----) and substrate side $(-)$.

Figure 6. (Color on line) Simulation results showing the effects of the hole diameters on the transmission ( - ) and reflection (----) spectra of various structures. Details of the structures are given in the headings of each plot. 
Figure 7. (Color on line) (a) Transmission and (b) absorption spectra simulated for structures with $P_{y}$ ranging from $300 \mathrm{~nm}$ to $900 \mathrm{~nm}$ and $D_{y}=0.5 P_{y}\left(\mathrm{E} / / P_{y}\right) ; P_{x}$ and $D_{x}$ are fixed to $400 \mathrm{~nm}$ and $200 \mathrm{~nm}$, respectively. Also shown are the theoretical $\omega^{+}(---)$and $\omega^{-}(-)$lines. 
Figure 1
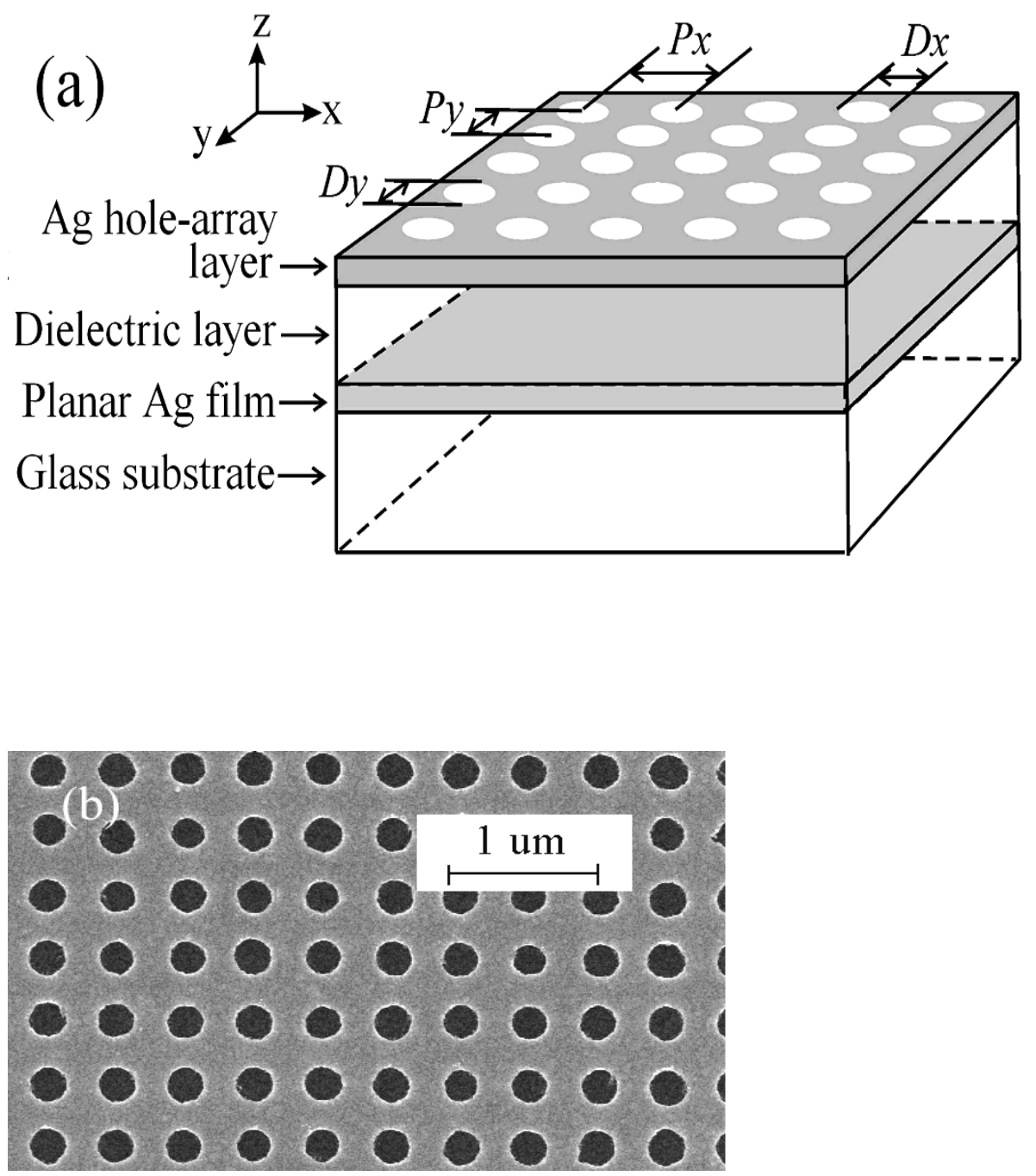
Figure 2
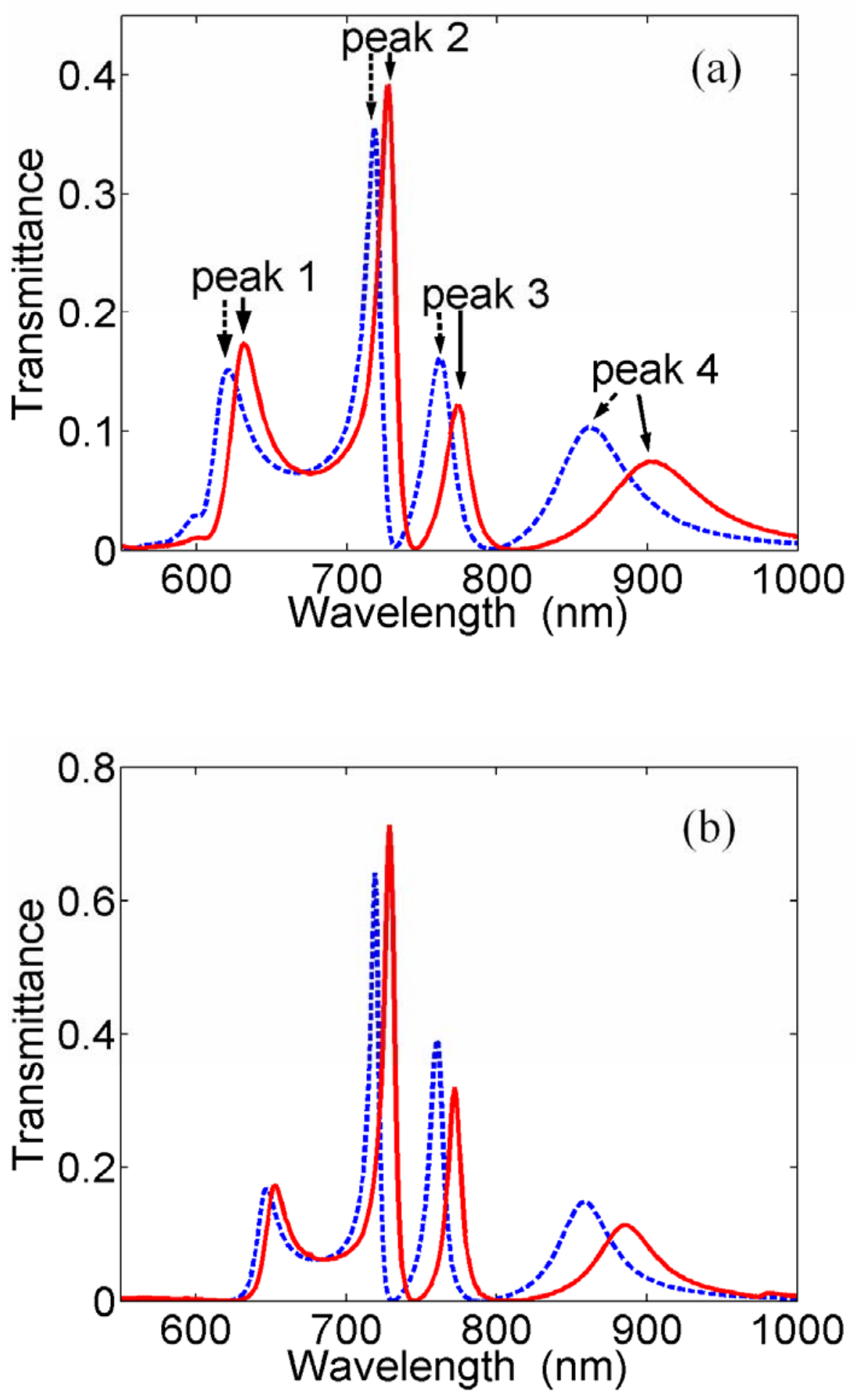
Figure 3

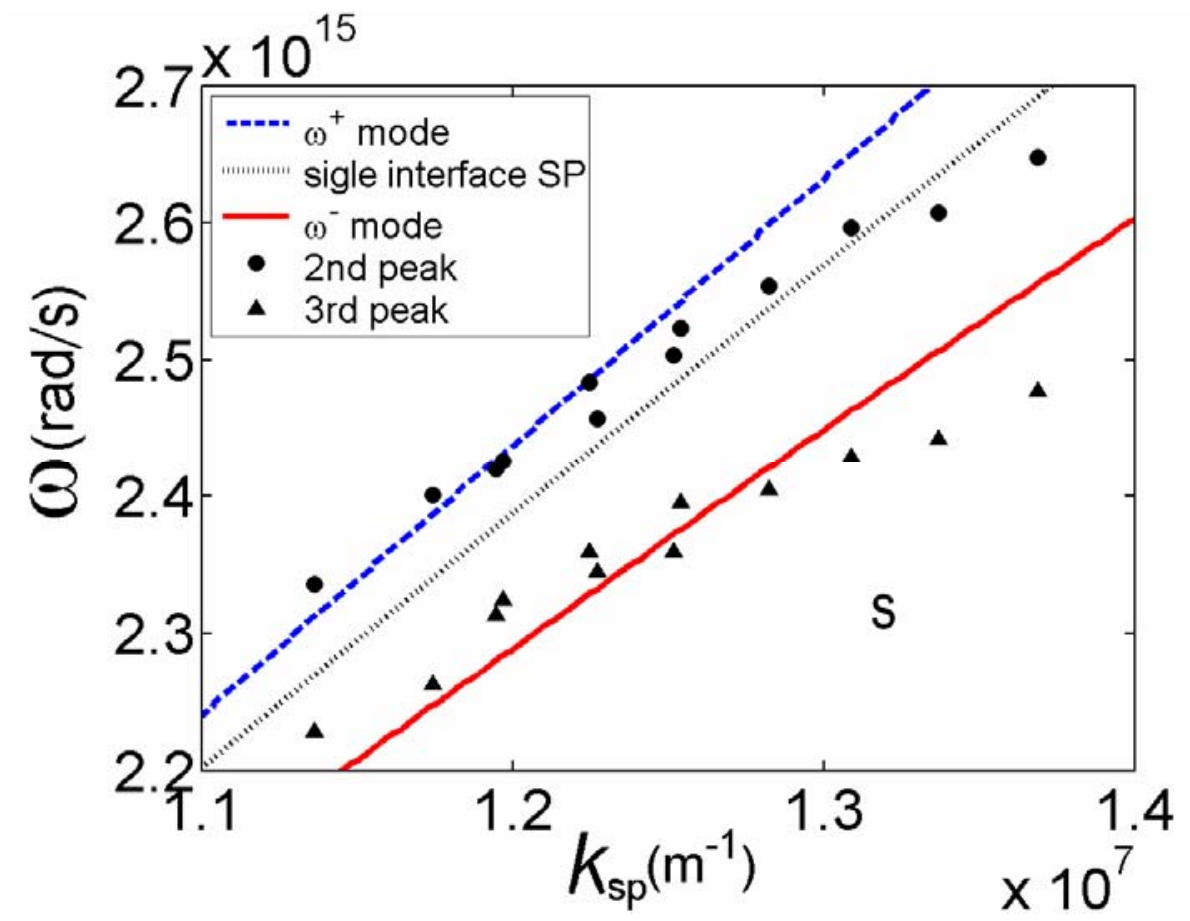


Figure 4

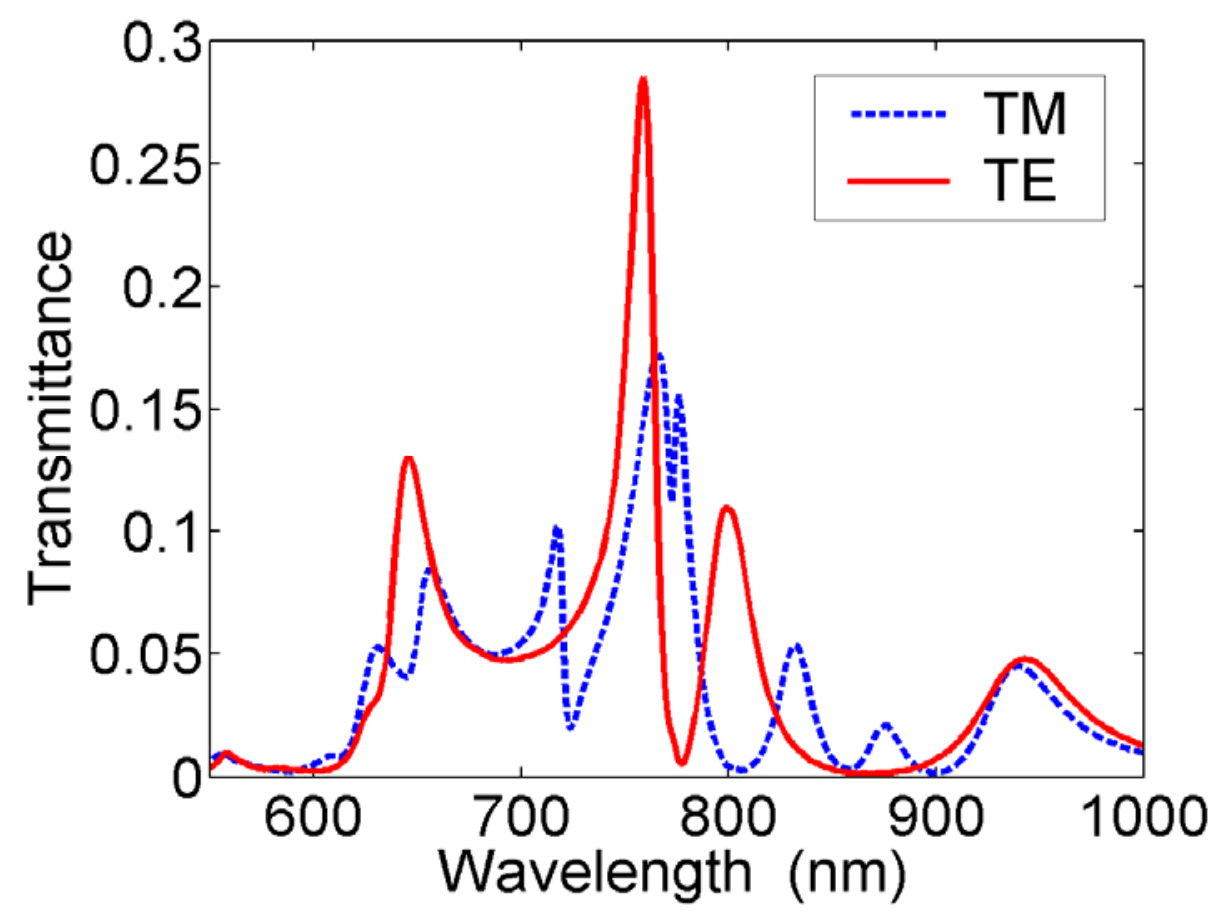


Figure 5
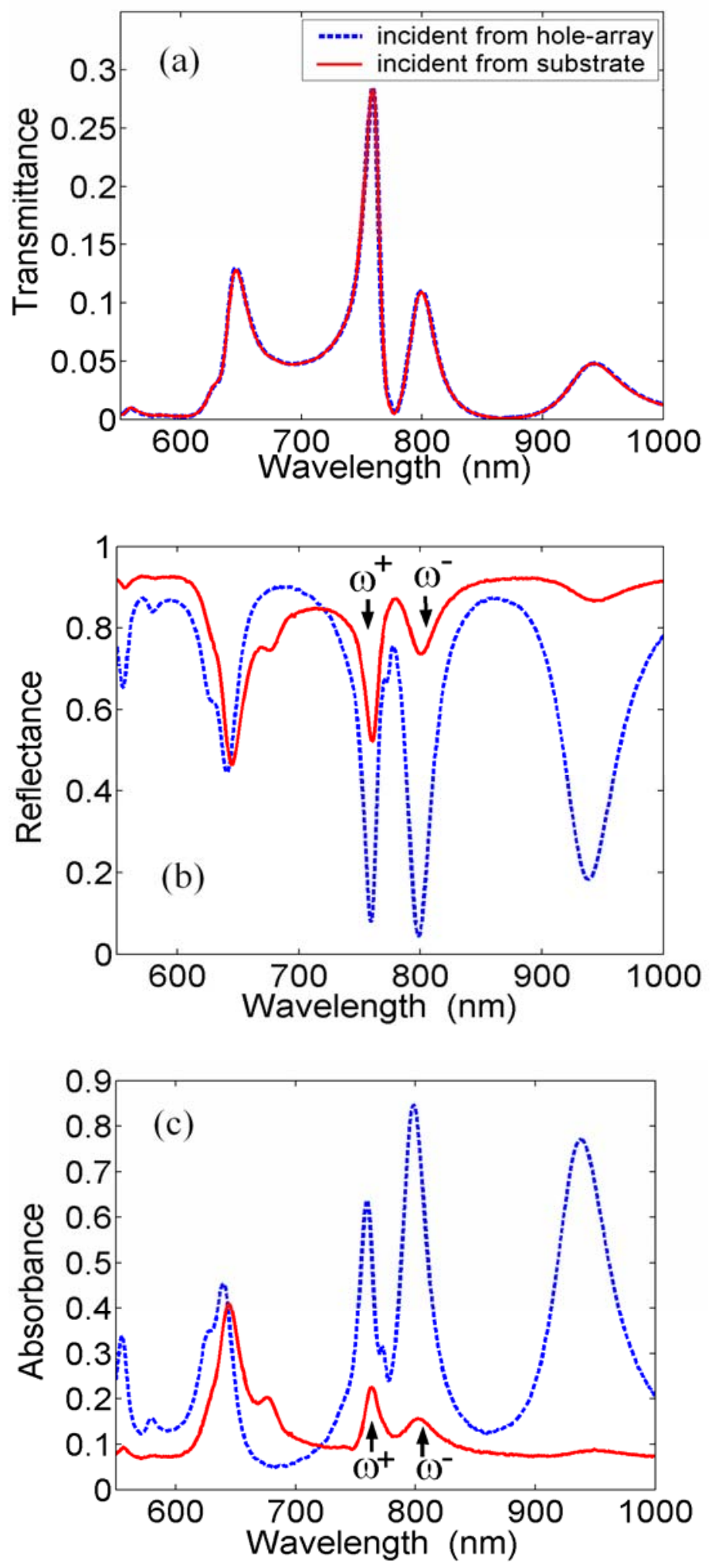
Figure 6
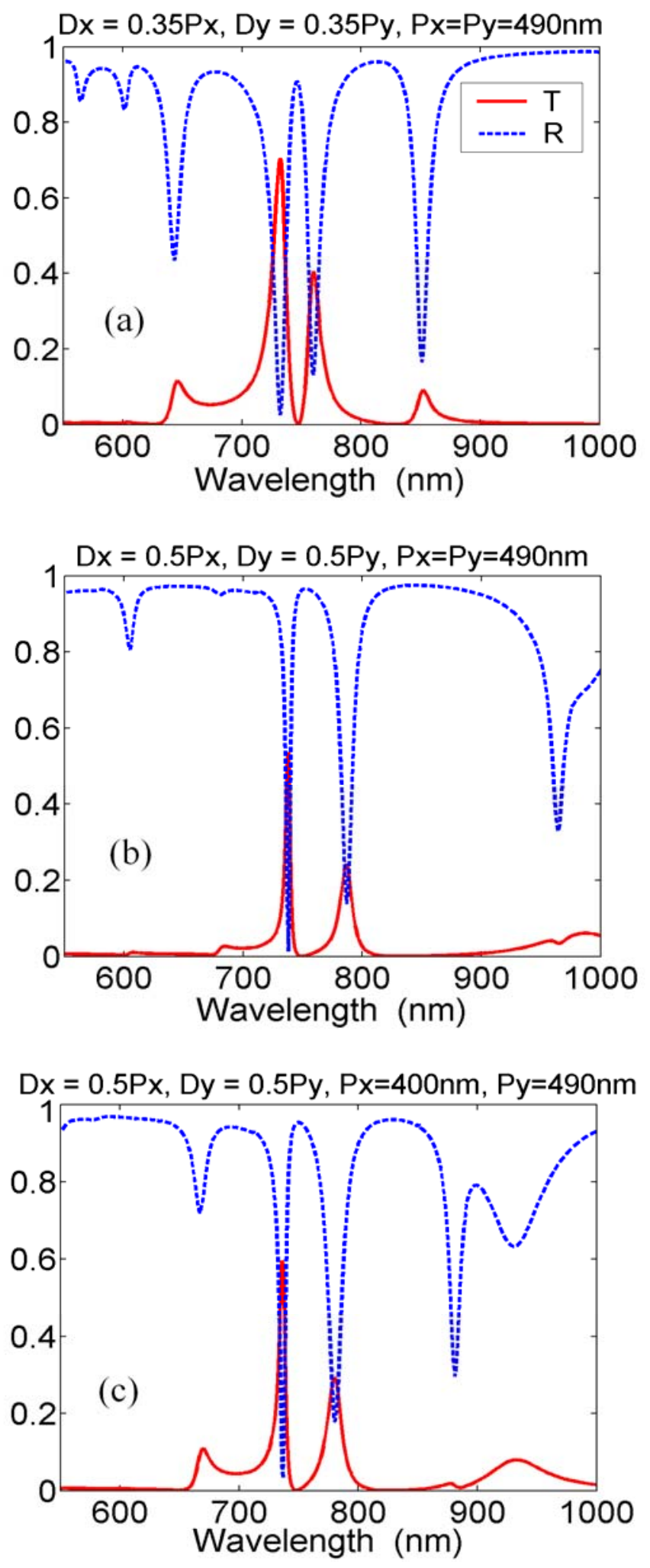
Figure 7
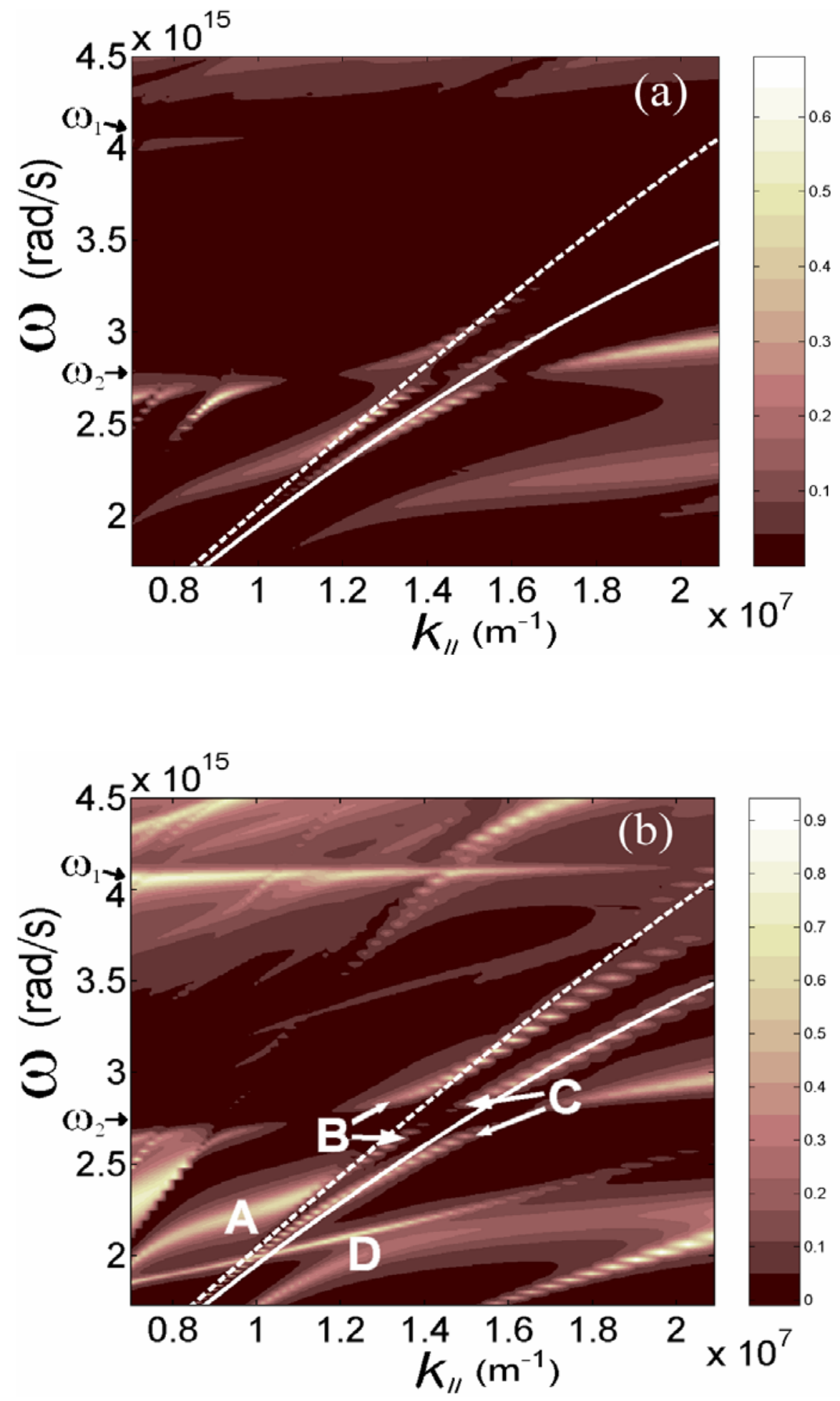